\title{
Heavy ion physics with the ATLAS detector
}

\author{
Helena Santos for the ATLAS Collaboration \\ LIP, Av. Elias Garcia 14-1, 1000-149 Lisboa, Portugal \\ DOI: http://dx.doi.org/10.3204/DESY-PROC-2010-01/233
}

\begin{abstract}
The ATLAS experiment will participate in the heavy ion program of the Large Hadron Collider (LHC), for which the main goal is to create strong interacting matter under extreme energy density and temperature conditions. Evidence from SPS and RHIC data suggests that in such extreme conditions matter undergoes a phase transition from ordinary hadronic matter to a plasma of quarks and gluons, the QGP. The large acceptance, high granularity calorimeters, silicon tracking detectors, and muon spectrometers assure that ATLAS can handle such a challenging program.
\end{abstract}

\section{Introduction}

$\mathrm{Pb}+\mathrm{Pb}$ collisions at the LHC are expected to produce a QGP with energy densities 2-3 times (or even more) larger than at RHIC, with larger initial temperatures (by a factor of 2) and longer lifetimes (the order of 1.5). To carry on this program, the ATLAS [1] experiment has a large detector acceptance, as shown in Figure 1, with full azimuthal coverage and 10 units of pseudo-rapidity, not taking into account the very forward detectors which will play a major role in the heavy ion program. In $\mathrm{Pb}+\mathrm{Pb}$ collisions, the detector is especially suited to study jets and photons. Concerning jets, the large acceptance and fine segmentation allows full reconstruction and a detailed study of their properties. For photons, the fine segmentation of the first layer of the electromagnetic calorimeter allows for separation of direct photons from those originating from $\pi^{0}$ and $\eta$ decays. This is a unique strength of the ATLAS detector at the LHC.

\section{Global observables}

The day-one physics will be devoted to establish the global features of the heavy ion program. The collisions centrality is characterized event-by-event by the impact parameter, $b$, which is intrinsically related to the geometry of the collision and so to the number of collisions and the number of excited participating nucleons. A strong correlation between these variables and the energy deposited in the ATLAS calorimeters is expected. Figure 2 (left plot) demonstrates how well ATLAS reconstructs the transverse energy over the whole pseudorapidity range in central collisions, $b=2 \mathrm{fm}$, taking into account correction factors for acceptance cracks and energy depositions by particles which originate from re-interactions in the detector material. Measurements of the inclusive charged particle density will be essential and few days of data taking will help establish which physics scenarios can be ruled out using the measured multiplicities [2]. The middle plot of Figure 2 shows the capabilities of ATLAS to reconstruct $d N_{c h} / d \eta$ in central collisions. The estimated errors are $10-15 \%$. 
HELENA SANTOS

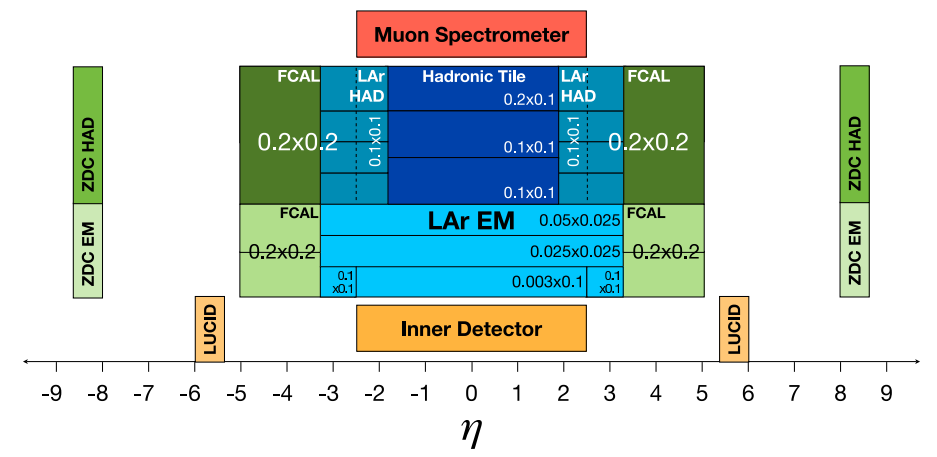

Figure 1: The pseudo-rapidity coverage of the various components of the ATLAS detector.

One observable that gives insight into the very earliest phase of the collisions is the elliptic flow, which arises when two colliding nuclei do not overlap totally. In such a case, the initial spatial anisotropy leads to a final state elliptical asymmetry in momentum space with respect to the reaction plane. The variable $v_{2}$ is the second Fourier coefficient of the particle azimuthal distribution with respect to the reaction plane and measures the elliptical shape of the particle's momentum distribution in the transverse plane. Figure 2 (right plot) shows $v_{2}$ as a function of the transverse momentum for mid-central events. Three methods to measure elliptic flow are applied to the simulated data - event plane, two-particle correlations and Lee-Yang Zeros [3, 4]. All three fail at the very low $p_{T}$ due the presence of fake tracks in this momentum range. The Lee-Yang Zeros method, however, shows the best performance and is less sensitive to non-flow effects.
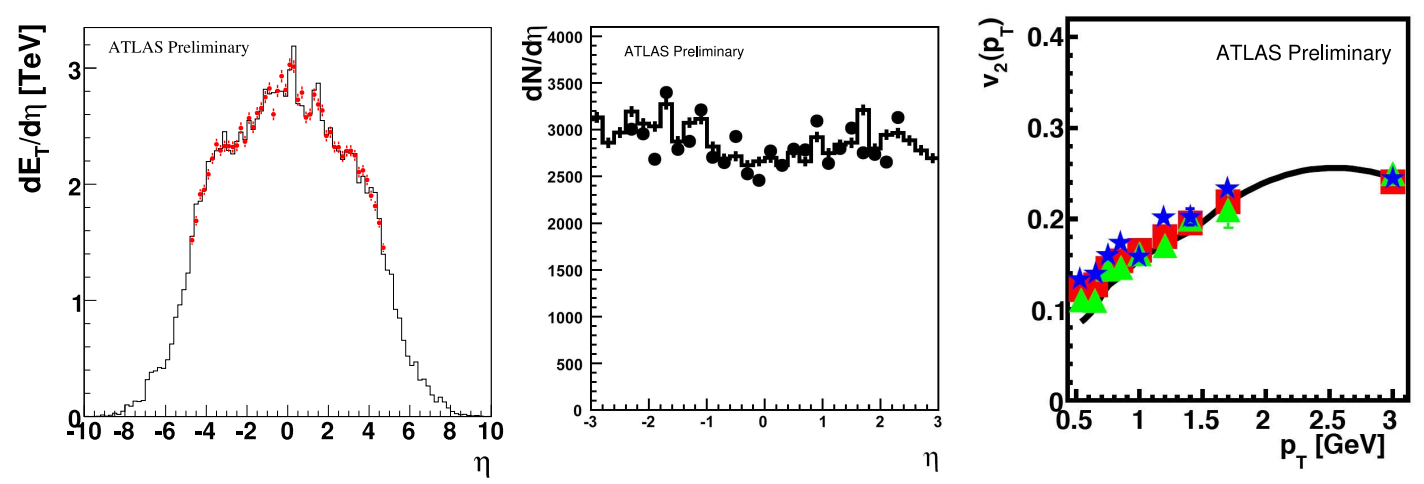

Figure 2: Left and middle: comparison of the reconstructed $d E_{T} / d \eta$ and $d N_{c h} / d \eta$ distributions (points) with the true distributions (histograms) for central events with $\mathrm{b}=2.3 \mathrm{fm}$. Right: $p_{T}$ dependence of the reconstructed $v_{2}$ from the event plane method (squares), two-particle correlations (stars) and the Lee-Yang Zeros method (triangles) for mid-central events, $b=7 \mathrm{fm}$. 


\section{HEAVY ION PHYSICS WITH THE ATLAS DETECTOR}

\section{Jets}

One of the most important topics of the LHC heavy ion program will be the jet suppression measurements $[5,6]$. Jets reconstruction in $\mathrm{Pb}+\mathrm{Pb}$ collisions is challenging due to the large underlying event. Thus, methods have been developed to subtract it by removing the averaged transverse energy, estimated far enough from the seeded jet signal, as well as to identify and reject fake jets $[7,8]$. Figure 3 compares the reconstructed jet spectrum with the input one, and the fake jet spectrum as a function of the transverse energy. Even without correcting for efficiency and energy resolution, the reconstructed spectrum matches the input one above 80 $\mathrm{GeV}$ quite well. The background at low $E_{T}$ is suppressed by two orders of magnitude. At 70 $\mathrm{GeV}$ the reconstruction efficiency is about $70 \%$, the resolution is approximately $25 \%$ and the background is negligible.

\section{Direct photons}

The design of the ATLAS electromagnetic calorimeter is optimal for direct photon identification [1]. As seen in Figure 4, the first layer has a very fine longitudinal segmentation along the $\eta$ direction, allowing to disentangle direct photons from $\pi^{0}$ and $\eta$ photon decays. This is a unique feature of ATLAS and rather important because direct photons are not affected by the strong interacting medium and so they can be used as a reference for jet suppression measurements [5]. Also the energy scale determination will benefit because the $p_{T}$-balance of di-jet correlations is of limited use due to the expected energy loss in the QGP and the fluctuations of the large underlying event. The left plots of Figure 4 show the reconstructed energy deposition in the strip layers as a function of the strip cluster size, $\Delta \eta$, for direct photons and

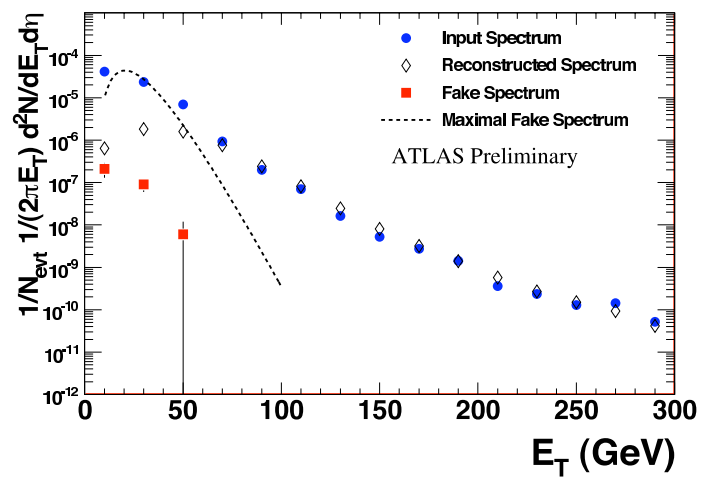

Figure 3: Input, raw reconstructed and fake spectra for cone jets in central $\left(d N_{c h} / d \eta=2650\right)$ $\mathrm{Pb}+\mathrm{Pb}$ collisions. The reconstructed spectrum is not corrected for efficiency and energy resolution. Dashed line represents the absolute fake jet rate from HIJING events prior to background jet rejection. for $\pi^{0}$ s embedded in HIJING central events.

The energy of a single photon is concentrated across a few strips with a single maximum at the middle, whereas a cluster from a $\pi^{0}$ decay is distributed across more strips.

\section{Heavy ions run in 2010}

The first heavy ion run at the LHC is scheduled for November and December of 2010. The CM energy will be $2.76 \mathrm{TeV} /$ nucleon pair, a factor 2 smaller than the nominal one. The peak luminosity will be 2 orders of magnitude smaller, $1-2 \times 10^{25}$. Taking into account the total inelastic $\mathrm{Pb}+\mathrm{Pb}$ cross section, 7.7 barns, an interaction rate of $80-160 \mathrm{~Hz}$ is expected. Some 


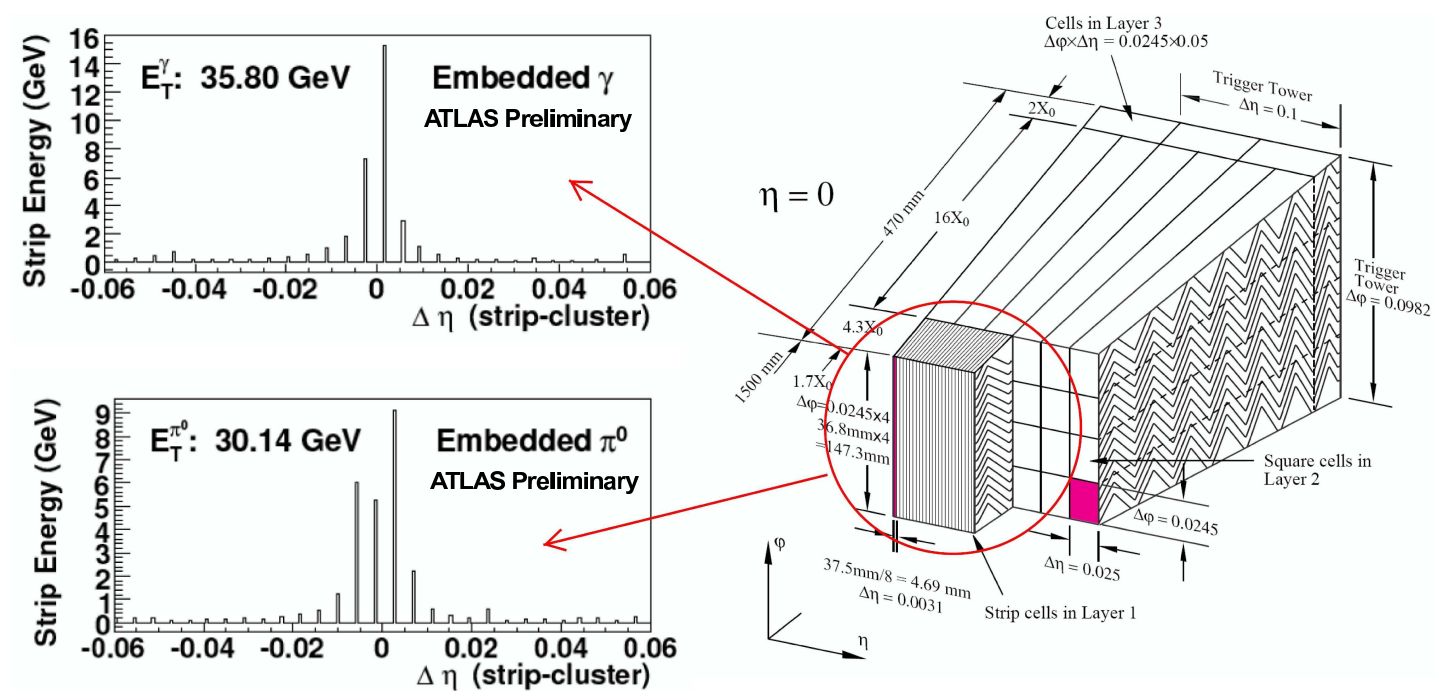

Figure 4: The reconstructed energy deposition in the strip layers around the direction of (top left) a single photon and a single $\pi^{0}$ (bottom left), both embedded in a central $\mathrm{Pb}+\mathrm{Pb}$ event. On the right the sketch of a barrel module with the different layers is shown, with the fine granularity in $\eta$ of the first layer being enhanced.

advantages of these low rates can be exploited, namely in triggering. In principle, depending on how large events will be, no Level-1 rejection is needed and the High Level Trigger will work in transparent mode. Beyond minimum bias, the Level-1 trigger will be used to find regions of interest concerning rare signals, namely jets, muons, and ultra-peripheral collisions. Even with low to moderate luminosity, $50 \mathrm{~Hz}$ written to tape will amount to two million $\mathrm{Pb}+\mathrm{Pb}$ events per day.

In conclusion, ATLAS is fully prepared to collect and analyze the first heavy ion data.

\section{Acknowledgments}

This work was supported in part by FCT, UE, COMPETE, and QREN under the contract Project CERN/FP/109323/2009.

\section{References}

[1] The ATLAS Collaboration, G. Add et al., JINST 3, S08003 (2008).

[2] B. B. Back et al. PHOBOS Collaboration, Phys. Rev. C74, 021902 (2006a).

[3] S. Wang et al., Phys. Rev. C44, 1091 (1991) and references therein.

[4] R. S. Bhalerao, N. Borghini, and J. Y. Ollitrault, 4 Phys. Lett. B580, 157 (2004).

[5] S.S. Adler et al. PHENIX Collaboration, Phys. Rev. C75, 024909 (2007a).

[6] J. Adams et al. STAR Collaboration, Phys. Rev. Lett. 91, 072304 (2003).

[7] N. Grau for the ATLAS Collaboration, J. Phys. G: Nucl. Part. Phys. 35, 104040 (2008).

[8] N. Grau, B.A. Cole, W.G. Holzmann, M. Spousta and P. Steinberg for the ATLAS Collaboration, arXiv:0810.1219. 